\section{$\underset{\text { hommes }}{\text { \& migrations }}$}

\section{Hommes \& migrations}

Revue française de référence sur les dynamiques

migratoires

1319 | 2017

Réfugiés et migrants au Liban

\title{
Les migrations
}

Grand angle sur un patrimoine méconnu en région Centre-Val de Loire

\section{Anthony Gauthier}

\section{(2) OpenEdition}

\section{Journals}

\section{Édition électronique}

URL : http://journals.openedition.org/hommesmigrations/4002

DOI : 10.4000/hommesmigrations.4002

ISSN : 2262-3353

\section{Éditeur}

Musée national de l'histoire de l'immigration

\section{Édition imprimée}

Date de publication : 1 octobre 2017

Pagination : 161-163

ISBN : 978-2-919040-39-1

ISSN : $1142-852 X$

\section{Référence électronique}

Anthony Gauthier, «Les migrations », Hommes \& migrations [En ligne], 1319 | 2017, mis en ligne le 01 octobre 2017, consulté le 08 janvier 2021. URL : http://journals.openedition.org/hommesmigrations/ 4002 ; DOI : https://doi.org/ERREUR PDO dans /localdata/www-bin/Core/Core/Db/Db.class.php L.34 : SQLSTATE[HYO00] [2006] MySQL server has gone away 


\section{LES MIGRATIONS : GRAND ANGLE SUR UN PATRIMOINE MÉCONNU EN RÉGION CENTRE-VAL DE LOIRE}

par ANTHONY GAUTIER, co-fondateur du site apostrophe45.

$\bigcup^{\prime}$ ne exposition à Orléans présente, jusqu'au 9 juillet, la diversité et l'ancrage des migrations en région Centre-Val de Loire sous un double regard : intime et historique.

La porte d'entrée de l'exposition Histoires de migrations, intimités et espaces publics en dit à la fois toute la richesse et l'originalité. D’emblée, le visiteur qui pénètre entre les murs de l'Hôtel CabuMusée d'histoire et d'archéologie, à Orléans, est plongé dans l'intimité quotidienne des migrants, comme s'il poussait la porte de leur foyer. De petits entretiens vidéo associés à une présentation d'objets personnels, hétéroclites, qui incarnent ou symbolisent le prolongement des traditions dans l'exil, accueillent chaque visiteur et le font entrer dans cette "intimité " sensible, plurielle, émouvante, surprenante. Témoin d'une vérité culturelle et familiale qu'elle seule est en mesure d'affirmer.

"On a voulu proposer un travail à la fois scientifique et sensible pour trouver les moyens de parler aux visiteurs. Ce n'était pas toujours facile de naviguer entre ces deux dimensions. Cela a été possible grâce au travail collaboratiffait pendant deux ans et qui débouche sur une collecte d'objets, d'entretiens, d'images, de photos, tout ce que les gens pouvaient nous offrir pour raconter leur histoire. Cette exposition montre ces trésors d'intimité que les gens ont chez eux ", souligne Hélène Bertheleu, sociologue, l'une des deux commissaires de cette exposition. Casserole, réchaud, seau à eau, boîte de safran, panier pour faire cuir le riz, canne, etc., disent ainsi, et pour la première fois, les " peines et les joies de l'histoire migratoire » dans la région. Une histoire cachée jusqu'à présent car réservée au seul regard des proches et de la famille. «Parfois, on a dû sélectionner des objets de façon à ne pas avoir une surreprésentation de certaines migrations. On a eu ainsi beaucoup de choses portugaises, et c'est normal puisque c'est la migration la plus importante, mais on a essayé de diversifier, et tout à la fin, nous sommes même allées solliciter des gens pour avoir des objets de la migration subsaharienne notamment », explique Hélène Bertheleu. Cette collecte inédite pouvait déboucher sur un autre danger, comme le concède la sociologue : celui de tomber dans « une vision individualisée de la migration, une collection de trajectoires individuelles mises les unes derrière les autres». Un écueil évité par la mise à disposition du public d'éléments historiques et sociologues qui "encadrent l'exposition et lui donnent tout son sens".

Outre le fait de porter un sujet méconnu sur la place publique, le grand intérêt de cette exposition repose donc sur ce double regard porté sur les migrations régionales, à la fois "sensible et historique ». Une approche inédite qui donne ainsi à voir une facette inattendue, de la région Centre-Val de Loire qui, si elle n'a jamais été une terre de grandes migrations contrairement à d'autres régions françaises, a accueilli de longue date une grande diversité de populations, et pas seulement venues d'Europe.

À ce titre, les migrations successives ont inévitablement façonné, même de manière discrète et par touches impressionnistes, le patrimoine culturel régional. «C'est un sujet très mal connu dans la 


\section{INITIATIVES}

région, qui véhicule beaucoup de stéréotypes à partir d'une grille de lecture souvent nationale", indique Hélène Bertheleu.

\section{La région Centre-Val de Loire au prisme des migrations}

Certes, les migrations ont été statistiquement moins importantes dans la région Centre-Val de Loire qu'en Aquitaine ou en région parisienne, par exemple, pour autant elles ont une histoire tout aussi ancienne. Qui sait, par exemple, que les Belges ont occupé le premier rang des migrations de la fin du XIX ${ }^{e}$ siècle jusqu'en 1921 et qu'ils consti-

tuaient près d'un tiers des

Un parcours mémoriel

qui s'exonère des seules

données chiffrées, sans les

oublier néanmoins, pour

rendre compte d'une réalité

patrimoniale humaine

touchante, diverse, riche et complexe. étrangers dans la région, soit environ 2 ooo personnes? Dans le seul département du Loiret, les Belges représentaient à cette période plus de $40 \%$ de la population étrangère. Ouvriers peu qualifiés pour la plupart d'entre eux, ils travaillaient sur les chantiers de chemin de fer ou comme saisonniers agricoles en Eure-et-Loir, par exemple. À partir de 1850, Allemands, Suisses, Italiens, Espagnols, Britanniques, Polonais, AustroHongrois, et Russes viennent s'installer en région Centre-Val de Loire, chaque migration apportant son ardeur au travail et son savoir-faire. Pour les Polonais, près de 500 recensés en 1850, les métiers exercés vont de la médecine à la typographie, en passant par l'enseignement des langues. "Cela démontre bien que les migrations ont été aussi européennes. II y a eu des migrations belges, italiennes, polonaises, ukrainiennes, du Maghreb, de I'Asie, de l'Afrique et d'Europe de l'Est plus récemment. On a toutes ces migrations. II ne s'agissait pas pour nous de faire une collection mais de montrer la profondeur historique et le fait que ces migrations constituent un véritable patrimoine local", explique Pôleth Wadbled, l'autre commissaire de l'exposition.
Ainsi, à l'heure où les questions d'immigration sont régulièrement instrumentalisées, utilisées à des fins politiques, l'exposition rompt avec l'image angélique d'un passé apaisé, d'un climat social toujours clément. "On pense toujours que les Italiens, les Espagnols ont vécu des migrations beaucoup plus harmonieuses parce que ce sont des migrations européennes. En fait, cet angélisme est démenti et opposé à un alarmiste au présent. II y a eu des chasses à l'homme, une xénophobie très forte et très violente à l'égard des Italiens dans le passé ", souligne Hélène Bertheleu. En particulier à la fin du XIXe siècle. «L La migration, ce n'est pas une pathologie sociale, on a tendance à oublier ", poursuit Pôleth Wadbled.

Les uns après les autres, les migrants ont naturellement contribué au développement de quasi tous les secteurs économiques régionaux. Mais il ne faudrait pas oublier les migrantes qui, elles aussi, ont joué un rôle notable : Écossaises, Irlandaises, Anglaises ont, en effet, été employées dans l'éducation et la domesticité au début du XXe siècle à l'échelle régionale. De nombreuses Irlandaises sont ainsi religieuses dans les couvents de Bourges, de Dreux et de Chartres.

L'exposition s'attarde également, sur la ville de Montargis, berceau prétendu de la révolution chinoise puisque la "Venise du Gâtinais» a accueilli, à partir de 1903, des étudiants chinois qui, pour subvenir à leurs besoins et payer leurs études, travaillaient à Hutchinson. Parmi eux, Teng Hi Hien qui deviendra sous le nom de Deng Xiao Ping, un dirigeant de la Chine communiste de 1978 à 1992. Cette même usine Hutchinson qui fournira du travail aux Russes "blancs» et aux Kalmouks qui fuient la révolution russe de 1917 . Et ils seront ainsi quelque 1200 à venir trouver refuge dans le Montargois jusqu'en 1926. Polonaises recrutées dans les fermes d'Indre-et-Loire dans les années 1930, travailleurs indochinois embauchés dans les usines d'armement pendant toute la durée de la Seconde Guerre mondiale, réfugiés espagnols majoritairement des femmes, des enfants et des personnes âgées - répartis dans les six 
départements de la région, les migrations obéissent souvent à cet impératif vital de trouver un abri loin de la répression et des menaces. Aprèsguerre, l'immigration en région Centre - maghrébine, algérienne et marocaine notamment - " reste modeste mais s'inscrit dans le grand mouvement de migration de travail vers la France" notent les auteurs de l'exposition.

\section{Un travail collectif au service d'une muséographie intime des migrations}

La capacité de cette exposition à rendre compte d'une réalité familiale par nature tenue hors des projecteurs publics, repose pour beaucoup sur la manière dont l'exposition a été construite : pendant deux ans, des groupes de travail hétérogènes, formés de personnes issues de la "société civile ", en tous les cas étrangères, au départ, à la genèse de l'exposition, ont travaillé autour de la même table. Une œuvre collaborative au sens strict du terme.

"La méthode était presque aussi importante que l'exposition elle-même, et il n'a pas été difficile de mobiliser la population en réalité. On a comptabilisé qu'au total, entre 100 et 150 personnes ont contribué directement à la fabrication de l'exposition, soit des personnes qui étaient directement présentes dans les groupes de travail, soit qui sont allées chercher elles-mêmes, dans leur propre réseau, des objets et des témoignages, ce qui a été très efficace ", précise Hélène Bertheleu. Bien entendu, le degré de participation a été inégal et fluctuant au cours de ces deux années. "Tous ceux qui ont construit avec nous cette exposition ne sont pas restés comme un seul homme pendant deux ans: certains ont pris part à tout le processus, d'autres ont été plus satellites. Mais peu importe, ce qui était intéressant était d'avoir sur chaque territoire un miroir de ceux qui se sentaient concernés par la question et de pouvoir aussi avoir une diversité d'acteurs; on a eu beaucoup des gens à double casquette, eux-mêmes personnellement concernés par l'immigration et puis en même travailleur social, écrivain public, etc. " Pendant plus de deux ans, donc, à Orléans, Bourges, Tours et Montargis, des chercheurs, des migrants, des descendants de migrants, des enseignants, des travailleurs sociaux, des artistes se sont installés autour des mêmes tables, pour collecter et classer des données, des souvenirs et des objets, pour enregistrer de petits films et pour reconstituer des parcours de vie. En sus, une archiviste, embauchée pendant huit mois, a écumé les archives municipales et celles issues du tissu associatif pour exhumer photos et données diverses. Et c'est cette intimité familiale reconstituée et inédite que l'exposition propose aux visiteurs de découvrir dans une scénographie multi-supports qui mêle des séquences audio, vidéo et des panneaux traditionnels sobrement conçus. Un parcours mémoriel qui s'exonère des seules données chiffrées, sans les oublier néanmoins, pour rendre compte d'une réalité patrimoniale humaine touchante, diverse, riche et complexe. Et qui met en lumière un autre héritage patrimonial que celui que la région Centre-Val de Loire promeut sans discontinuer: celui de ses châteaux et de sa Loire.

\section{Pour aller plus loin :}

- Guillaume Étienne, Histoires de migrations, Intimités et espaces publics, Tours, Presses universitaires François-Rabelais, 228 pp., 19 euros. 